\title{
Diskussion: Parteienfinanzierung
}

\author{
Günter Frankenberg \\ Wider den Rechtsopportunismus \\ Anmerkungen zur Situationsjurisprudenz in der \\ Parteienkrise
}

I.

Wo politischer Opportunismus regien, kommen Verfassung und Gesetz nicht zu ihrem Recht. Einer politischen Klasse, die dem Diktat des eben Günstigen folgt, werden Rechtsnormen, die Macht begrenzen, Pflichten staruieren oder Kontrollen vorschreiben, zur lästigen Fessel. Der Opportunist lässt nichts unversucht, sie abzustreifen oder, wo das nicht möglich ist, zu lockern. Ans Recht hält er sich nur, wenn und solange es Vorreile versprichr. Im übrigen ziehr er es vor, im Schattenreich von Macherhale und Eigennucz je nach Bedarf heimlich vom Wein des Illegalen zu kosten, öffentlich gleichwohl das Wasser des Rechrsgehorsams anzupreisen. Selbst dann, wenn seine Doppeizüngigkeit offenbar, wenn die mühsam maskierte Illegalität zum Skandal wird, kann sich der Opportunist zum Recht nicht bequemen. Beruft sich an dessen star aufs Ehrenwort, bitcet um Entschuldigung, spricht von „Dummheita oder $n$ Ärgernis* und ziert sich in wohlinszenierten öffentlichen Zerknirschungstänzen vor den rechtlichen Sanktionen. Insgeheim hoffend, das staunende bis empörte Publikum der Rechrsgenossen werde sogar dann noch der Umgehung des Rechts applaudieren und dem ertappten Opportunisten willig in den Morast einer Moral folgen, deren biegsame Regeln ohnehin das Schicksal von Verfassung und Geserz teilen. Denn auch dorr, nein, gerade dort in Nebel des Außerrechelichen triumphiert der kacegorische Imperativ des Opportunismus: Verhalte dich so, dass die Maxime deines Handelns sters ein für dich günstiges Ergebnis garanciert.

Als bloße Texte bleiben Verfassung und Gesetze kraftlos. Sie kommen zur Geltung, wenn sie von einer wachsamen Bürgerschaft getragen, von verfassungs- und geseczestreuen Amsträgern befolgt und von ihren Interpreren nach den Regeln juriscischer Kunst ausgelegt werden. Eine Demokracie stellt dafür ein denkbar günstiges Umf́ld bereit. Wie leicht sie in eine Krise gerissen werden kann, zeigt schlaglichtartig das Finanz-Debakel der CDU. Bedrohlich ist weniger die Selbsidemontage eines Bimbes-Kanzlers oder die erst normative, dann vielleicht materielle Verarmung einer "Volkspartei๔. Nein, zur Bedrohung für die Demokratie wird, wenn eine politische Klasse sich vorwerfen lassen muss, zur zivilen Desertion anzustiften, zur Fluchr der Bürgerschafr aus der politischen Aktivität. Aus gegebenem Anlass, der zunächst als das "System Kohl $\ll$, dann auch als die "Methode Kanther $\alpha$, schließlich als Parteispenden-Affäre bezeichnet wurde, und im eigcntümlichen Flugverhalten von sozialdemokratischen Amtsträgern zeichner sich der nächste Anlass ab, sollen hier zwei Klagen geführt werden. Eine gegen das antidemokratische, gestöne Verhälenis der an schwarzen Kassen, illegalen Spenden und Lügengebäuden zu ihrer Verdeckung betciligten Manipulatoren zu Grundgesetz und Parteiengesetz. Eine andere gegen professionelle Rechesinterpreten, die statt Distanz zum politischen Opportunismus 
eine neue Variante der - in Weimar vom Staatsrechtslehrer Hermann Heller kritisierten - Situacionsjurisprudenz« kultivieren.

II.

Die Störung aut Sciten des Bundeskanzlers a.D. Helmut Kohl hat die tageszeitung auf den Punkr gebracht. Schuld an allem sei Rita Süßmuth, die es als Präsidentin des Deurschen Bundesrages unterlassen habe, Helmur Kohl vor Antritt seiner Kanzlerschaft das Ehrenwort abzunehmen, er werde die Ver\{assung wahren. Leicht fahrlässig hatce sie darauf vertraut, das sei mir der Abnahme des Amiseides zu erledigen, der da unter anderem lauret: »Ich schwöre, $\mathrm{da} B$ ich ... das Grundgeserz und die Gesetze des Bundes wahren und vercidigen, meine Pflichten gewissenhaft erfüllen und Gerechtigkeit gegen jedermann üben werde.« Vier Amtszeiten, also viermal geschworen. So wahr ihm Gott helfe. Das reichte nicht hin, den drei "Körpern des Königsw in Gestalt des Parteivorsizzenden, Abgeoróneten und Kanzlers den ihnen gebührenden Pląz im Verfassungsystem anzuweisen.' Nämlich unter dem Grundgesetz, das in Art. $21 \mathrm{I}_{4}$ und operationalisert in den $\$ \$ 23$ ff. PartG den polizischen Parteien zur Pflicht macht, über die Herkunfr und Verwendung ihrer Mittel öffentlich Rechenschaft zu geben. Auch unter den Verhaltensregeln, die der Bundestag auf Befehl des Abgeordnetengesetzes beschloss, zu denen die Pflicht von Abgeordneten gehört, Spenden ab 10000 DM anzuzeigen. Über Verfassung und Gesetz steht einsam und allein die Bürgergesamtheic als demokracischer Souverän. Für sich genommen bewegen sich Bürgerinnen und Bürger, ob sic denn öffentliche Ämter verwesen, von Berufs wegen dic Verfassung interpretieren oder einer anderen ehrenwerten Beschäfigung nachgehen, ausnahmsios unter dem Grundgesetz und den einfachen Geserzen. Das zur Erinnerung an den Bürger Kohl.

Ein ehemaliger Bundeskanzler verweigert hartnäckig die Angabe mutmaßlicher Geldspender unter Berufung auf ein vermeintliches Ehrenwort. Mehr noch, er behauptec, nur seine Pflicht getan zu haben. Das beweist, er hat den Sichrkontakt zum Recht verloren. Er hätte vortragen können, sich angesichts der gegen ihn erhobenen strafrechtlichen Vorwürfe nicht selbst belasten ¿u müssen. Was wohl nicht fein genug war. Pflicht und Ehre mussten her, ihn cin für allemal vom Recht zu dispensieren. Den rechtswidrigen Rest sollce das Eingeständnis von "Fehlernu entsorgen. Der Beifall, den törichte Pfeffersäcke in (rechts-?) Freien Hansestädten allzu großzügig dazu spenden, darf nicht täuschen: Ein solcher Dispens ist im demokrarischen Rechrsscaat nicht zu haben. Den Unbclehrbaren haben unlängst insbesondere der ehemalige Verfassungsrichter Ernst-Wolfgang Böckenförde ${ }^{1}$ und der Philosoph Otfried Höf$f^{s}$ energisch und mir eindrucksvollen Argumencen belehrt, die Elire beinhalte keinesfalls wein vom öffentlichen Rechtsgesetz unabhängiges, im Konkurrenzfall ihm sogar überlegenes Geseczu. Im Gegenteil, ein solches Ehrenwort, unterstellt, es sei gegeben worden, so Höffe, unterlaufe die "Minimalscufe des schon aus der Ancike bekannten,

\footnotetext{
1 Zur l)okırin von den xwet Korpern des englischen Königs vgl. E. Kantorowicz. The King's Two Bodies. Eine Studie zur politischen Theologic des Mittelaleers (1957), de. Übers. München 1990.

2 E. W. Bockenförde, Dic Krisc unserer Demokratic verlangt eine Ruckbildung des Parkcicnstuatcr, FAZ von I4. 2. 2000. Vgl. auch H. H.v.Arnim, Wie ausder Krise cine Chance werden kain, FAZ vom 1।. 2. 2000; H.J. Vogel, Die Position der Wähler gegenüber din Pareien stärken, FA7. rom 19.2.2000 und R. Leichx, Dic Sir.afe komme erse noch, DIE ZEIT vom i7. 2. 2000.

3 O. Höffe, Ehre, wem Ehre gebühr, FAZ vom 3-2. 2000.
} 
aufgcklären Ehrbegriffs: dic Rechtsgesinnung* und widerspreche dem höherrangigen Versprechen. Gegeben mit dem Amrseid.

Die Parteifreunde, von ihrem ehrpusseligen Ehrenvorsizzenden im Stich gelassen, bemühten sich um Schadensbegrenzung. Als eben sich die Spitze des Eisbergs zeigte, wurden sie nicht müde, im Eingangsstatement zu jedem öffentichen Auftritt die historischen Leistungen des inkriminierten Ex-Kanzlers zu betonen. Verlegen folgte im Nachgang, nach der Salami-Taktik, scheibchenweise das Eingeständis von Fehlern, Ungenauigkeiten und Ungeschicklichkcicen. Eine bei Normbrüchen eher ungewöhnliche, opportunistische Semantik und Vorgehensweise. Im Rechtsstaat wird üblicherweise zunächst gefragc, exakr welche Rechtsverlerzung vorliegt. Dann, ob sie sich möglicherweise rechtfertigen lässt. Und schließlich, ob den Angeschuldigten ein Schuldvorwurf triff, oder ob er glauben durfte, er habe nichrs Verbotenes geran. Erst ganz am Ende, bei der Bemessung der Sankrionen, kommen die Verdienste des Täters und sonstige mildernde Umstände ins Spiel. Für diese Prüfungsfolge spricht rechtsstaaliche Ökonomie. Sie umzukehren, legt die Vcrmutung nahe, hier solle vorschnell entlastet, abgewiegelt, entschuldigt werden, hier werde ein Sonderrecht reklamiert. In einer Gleichheitsgesellschaft gerät das allenal zu einem heiklen Unterfangen, das die Begründungslast dramacisch erhöhr und daher die Becrofienen regelmäßig überfordert. Was auch geschah. Kaum waren die Geduldspolster der Gerreuen durchgescheuert, da ändernen sie die Losung von "Rettet das Image des ehemaligen Kanzlers" in „Rectec die Partei und am Ende "Rette sich, wer kann «. Was danach einsetzte, als Opportunität einen Kurswechsel dikticrte, wird als die seir Kant »brutalstmögliche Aufklärunga auf deutschem Boden in die Geschichte eingehen. Diese wird, so viel ist fast schon gewiss, im Sande der Beratungen parlamentarischer Uncersuchungsauschüsse verlaufen.

\section{III.}

Zur zweiten Klage. Sie richter sich gegen einige professionelle Deuter des Rechts. Genaver: Lehrer des Staatsrechts. Wo Verfassungsbruch sich in den Mantel von Pfliche und Ehre hülir, wo sich die Autoren von Gesetzen nicht mehr als deren Adressaten begreifen, wo das Parteiengesetz vielmehr als Einladung zur fröhlichen Übertretung missverscanden wird, dort sollten sich, wenn alies mit rechten und rechisstaatlichen Dingen zugeht, die Vertretcr des Faches Staats- und Verfassungsreche dem Geist des Opportunismus widerserzen. Ihnen fällt eine dreifache Aufgabe zu. Nicht gewöhnungsbedürftig dürfre für sie sein, als Lehrer und Anwälce vornehmlich des Grundgeserzes, aber hier auch des Parteiengesetzes aufzurreten. Ein dritter, höchst heikler Auftrag gesellc sich hinzu, wenn in besonders schwierigen Fällen Therapeuten eines beschädigten Rechtsbewußtseins gefragr sind.

Vielen Rechiswissenschafflern hat der Finanz- und Rechrs-Skandal ganz offensichtlich die Sprache verschlagen. Sie bestaunen die Ermittlungsarbcit der Medien, delegieren an diese die Aufgabe, das Grundgesetz zu verteidigen, und warten ab, bis alles, aber auch alles auf dem Tisch ist. Für die abschließende juristische Beurteilung sicherlich unerlässlich. Zu befürchten ist, dass sie und wir die Basis des Eisbergs nie nicho einmal unter der fragaürdigen Assistenz des Stasi - zu Gesicht bekommen werden. Auch geht es nicht um ein richterliches Urteil, bei dem der Zweifel schweigen soll. Sondern darum, einer opportunistischen Rechts- und Verfassungspraxis das Wasser abzugraben. Nicht wenige Verfassungsrechtler sind diesem Ruf gefolgt. Einige haben in pädagogischer Absiche an dic rechtlichen Grundlagen des Pareien- 
staats erinnert. ${ }^{+}$Andere haben sich nicht gescheut, wie Böckenförde von Demokratieund Parteienstaatskrise zu reden und der Erosion von Grundgeserz und Demokratie entgegenzuarbeiten.'

Kritische Fragen provozicren allerdings jene Fachverteter, die sich auf eine paradoxe Intervention kaprizieren. Der nicht unbegründeten Befürchnung, die Poliriker könnten sich gewohnheitsmäßig aufs Aussitzen verlegen und die Ermüdung des öffentlichen Interesses abwarten, treten sie mit einem Abwieglungsdiskurs zur Seire. Tiefer hängen, ist ihre Devise. Der Sorge, der Rechtsopportunismus eines Bundeskanzlers und seiner Klassenkameraden körnnte Vorbildwirkung entfalren, wollen sie mit clownesken bis schneidigen Abwehrargumenten jede Berechtigung stehlen.

Der Reihe nach. Als einer der ersten warf der Münchner Staatsrechaler Ruperr Scholz den Fehdehandschuh in den Ring. Mir nachgerade jugendliclicm Ungestüm im Angriff die Verteidigung suchend, wie es einem Verteidigungsminister a.D. fromms, sprang er dem bedrängten Ehren-Kanzler bei. Bis zum Bewcis des Gegenteils wollte er diesen keiner kriminellen Handlungen geziehen wissen. ${ }^{6}$ Was an sich niche zu beanstanden ist. Doch wollte er es damit nicht bewenden lassen. Die Empörung des Aufrechren ließ ihn (derzeir noch) unbewiesene und wegen des Schweigens der Berelligcen auch kaum beweisbare Vorwürfe herbeiargumentieren. Von Besrechlichkeit und Vorteilsannahme kann in der Tat noch keine Rede sein. So versuchte er, allzu durchsichrig, abzulenken vom eigentlichen Ziel der öffentlichen Actacken. Nämlich einmal von Äußerungen namhafter Strafrechtswissenschaftler, denen sich ein Anfangsverdacht scratrechtliclier Untreue zu Lasten der Partei aufdrängr. Dann und vor allem vom selbstherrlichen Umgang cines Bundeskanzlers mit dem Grundgesetz. Als Heerführer ruft Scholz alle wseriösen Geisteru herbei, sich mit ihm gegen den »Rummel«zu stemmen. Er serze ernücheernde Anführungszeichen (vor und hinter die „CDU-Spendenaffäre«), bekräftig mutig die Legitimität von Parteispenden, welche kaum bestritten wird, ${ }^{7}$ und weisı den Untersuchungsausschuss des Bundestages schneidig in die Schranken. Kein Wort zum Amtscid, kein Wort zur vertassungsunmitcelbaren Verbürgung des Transparenzgebots. Gewiss, am Anfang und Ende der wütenden Tirade gegen das Gebräu von Verdächrigung, Denunziacion und Verumglimpfung " kommi Scholz auf Verscöße gegen das Parteiengesecz und *Fehler z zu sprechen, die saufgeklärt und reparierta werden müssen. Und bestreitet nicht, dass die CDU gegebenenfalls die Konsequenzen zu tragen habe. Doch geht für ihn die Gefahr in erster Linie von den unmäßigen Kritikern aus, die sich mebenso an unserer Demokratie wie an unserem Rechtsstaat vergehen . Auch so kann man die Dinge sehen.

Wo Scholz das grobe Schrot nichr schcut, beflcißigt sich sein emeritierter Fachkollege Gerd Roellecke der milden Ironie und spricht in schiefen Bildern. ${ }^{*}$ Von den crwähnten ndrei Körpern des Königsa kommen, ohne Ironic, zwei bei ihm vor. Den Amtseid sieht er wohl, allein für nicht gebrochen hält er diesen. Kohl habe als Kanzler geschworen, jedoch als Parreivorsiczender kassierz. Der Körper des Parteivorsizzen-

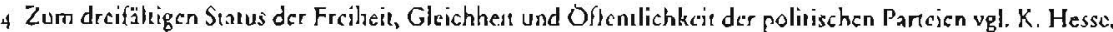

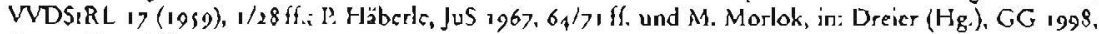
Art. 21 Rn. 4 Gil.

s Zu den aktucllen Problemen vgl. M. Morlok, Spenden-Rechenschafı-Sanktionen, NJW 2000,761 ff. Siche auch dic Wortmeldungen yon Vogel und v. Arnim (Fn. 2). Zurückhaliend Bull, Dic Alternative zum System Kohl, FAZ vom 23. 2. 2000, und, sich auf die Erlauterung der Rechsslage beschränkend. H. J Klcin, Momentanes Ärgernis, kein grundsätzliches Übel, FAZ vom 14. l.2000, sowie K. Srern, Warum

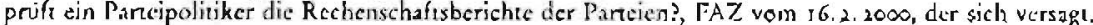
"Geschehencs (zu) bewerten* und sich auf Relormubeglegungen konzentriert.

6. R. Sehol7. Schaden fur Rechisstiar und Demoknic (Rubrik:Fremde Federn), FAZ vom 32.12. 1999

7 Vgl. BVCrfGE 73. $70 / 86$ und 85, 26, $/ 278$ !f. sowic Morlok. (Fn. 4), 763 f. m.w.Nachw.

8 G. Roclecke, Politiker werden mit Bargeld bervorlen wie anderc Leute mir Dreck, FAZ von 20, 1. 2000. 
den, so dürfen wir folgern, war bei der Eidesleistung nicht zugegen, hat also keine Hand zum Schwur crhoben. Der Körper des Kanzlers war - wie praktisch - bei der Annahme der Geldspenden nicht präsent. Kohl can do no wrong. Jedenfalls solange er sein Häuschen in Oggersheim eingeschossig "hälc und ith der Vorwurf der persönlichen Bereicherung nicht trifft. Verbinder man diese Bungalow-Theorie der Rechenschaftspflicht mit der Merhode der Körpertrennung, so springt eine bemerkenswcrte normacive Spende für Amts- und Mandacsträger dabei heraus. Der Amtseid wird cncwertec, das Grundgesetz bleibt außen vor. Künfrigen Kanzlern und Ministern, die ebenfalls Gesetzestreue auf ihren Eid nehmen müssen, ist dringend anzuraten, sters als Parteimitglieder, nicht aber als eingeschworene Amtsträger Bares oder Schecks in Empfang nehmen.

Es kommt noch besser. Von der verfassungs- und kanzlerschonenden Methode der Trennung lässt sich Roellecke, ganz konsequent, auch bei der Deutung der Rechenschaftspflicht leiten. Er nabelt sie von ihrem demokracischen Kontext ab und reduziert sie fröhlich aufs Finanzielle. Ergebnis: Wenn die Partei für ihre Pflichtverletzung gezahlt hat, ist die Sache ausgestanden. Spender und Verwendung der Mittel können dann im Dunkeln bleiben. Denn "um Aufkłärung geht es dem Gesetz nichr $\alpha$, Bedauerlich nur, dass Bundesverfassungsgerichr wie auch Kommentatoren des Arr. 2 I GG das ganz anders sehen als Roellecke.? Die Rechenschafuspfliche soll Vorsorge treffen, dass die Öffentlichkeit Kenntnis über die Herkunft der Mitcel erhält und erkennen kann, wer hinter den Parteien steht. Wer ist hier mit normativer Blindheit geschlagen, dass er die republikanisch-demokratischen Konnotationen des Transparenzgebots nicht zu erkennen vermag?

Roelleckes Situationsjurisprudenz lässt sich erklären, wenn wir seine Perspektive einnehmen. Er führt uns in den Secondhand Shop systemtheoretischer Bauteile, wo ihn vornchmlich die Ausdifferenzienung von Politik und Wirtschaft interessiert. Er bieter eine Metaphorik an, die uns nahelegt, das Grundgesetz etwa als eine Straßenverkehrsordnung für Politik und Wirtschaft und das Partciengesetz als Betriebsordnung einer Kraftahrzeug-Zulassungsstelle zu betrachren. So gesehen, ist es geradezu bedrängend plausibel, die Regeln der Rechenschaftsicgung mit Roellecke als "Parceien-TÜJ « zu bezeichnen. Sie tabuisieren die Trennung von Politik und Wirtschaft. Wer mit Geldzahlungen politische Geschäfte machen will, überschreitet eine unsichtbare Grenze und bricht ein Tabu. Wer als Politiker Geld annimme ebenso. Tabuverletzung statt Verfassungsbruch, damit lässt sich leichter leben.

Der kanzlerschonenden Grundgesetzlehre setzt Josef Isensce, als einer der bedcutendsten und wormächrigsten Staatsrechrslehrer vorgestellt, die Krone auf. ${ }^{10}$ Eben noch sich als Präzepror der Besonnenheit in Pose werfend, lässt er alsbald die Peizsche knallen. Schneidend geht sie auf den Präsidenten des Bundestages nieder, den Roellecke fast fürsorglich vorm "Falschparken gewarnt hatte. nMir der Urteilsgewissheit des Rechrslaien a habe eben jener Thierse, so Isensee. Helmut Kohl vorgeworfen, "Recht und Gesetz gröblichst missachtet« zu haben. Einmal in Schwung zieht der Bonner Staatsrechislehrer gegen alle Teilnehmer des "semantischen Überbierungswetrbewerbs" ins Feld, die "Verfassungsbruch * wittern, wo doch nur eine einfache

\footnotetext{
9 Zu den Beraungen des Parlamentarischen Rates vgl. v. Drocmming/Füßlein/Maız, JöR n. F i (1951), 207: zur Begröndung des Regicnungsent wurfs cincs Partciengesetzes betreffend dic Umsctzung der Rechenschafispflicht vgl. BT-De. III/1909. 28 und den Berichi des Innenausschusses des Deueschen Bundestages 7.um Entwur cinas Gesetzes zur Ncuordnung der Parteicnfinanzicrung, BT-Dr. 10/697, 4. BVerfGE 20, s6/ros; s2, 63/86L; 85, 264/319 \&f; Prcuß, AKGG 2. Aufl. (1989), Arl. 21 Rn. 74; v. Münch, in: v. Munch (Hg), GG II (1983), Ar. 21 Rn. 48 ff; Jarass/Picroth, GG 3. Aun. (199s). An. 21 Rn. 2 iff; Morlok, in: Dreier (Hg.), GG II, Art. 21 Rn. lasff; Ipsen, in: Sachs (Hg-), GG 1999, Art 1 I Rn. 106.; Scifert, Dic politischen Pancien im Recht der Bundesrepublik Deurschland (1975), 317.

$10 \mathrm{~J}$. Isensce, Das System Kolyl-Das System Rau, FAZ v. 28. 1. 2000.
} 

Parteien mussen Rechenschaft geben? Ja schon, aber Isensee weiß, wie das eigentlich gemeint ist: nur als programmatische Vorgabe, als Auftrag an den Gesetzgcber. Folglich seien Parteien zu Spendenpublizität verpflichtet, nur wenn und soweit das Parreiengesez sie vorsehe. Und dieses richre sich allein an die Parteien. Isensee schließt messerscharf: Weder der kanzlerhafte noch der parteimäßige Körper Kohls falle unters Parteiengesetz. Allein scin dritter Körper habe gesündigt und die Verhalıensregeln für Abgeordnete ( $\$ 44_{4}$ II Nr. 3 AbgG iVm $\$_{4}$ II Verhaltensregeln) übcrtreten.

Die Ansicht ist apart. Ihre Begründung erst rechc. Isensee zitiers mit großer Geste "Staarspraxis und Scaarsrechtslehre als Kronzeugen seiner Verfassungsdeutung herbei. Freilich werden längst nicht alle für ihn aussagen. Am nächsten kommt seine Auffassung einem obiter dictum, formuliert vom Bundesverfassungsgericht in der Entscheidung zum Verbot der Sozialistischen Reichspartei. Das war 1952." Später wird die Zeugenaussage der Verfassungsrichter deutlich ungünstiger. Mit vergleichsweise ausführlicher Begründung sprechen sie seit 1966 von einem nan die Parteien gericheeten Gebot, über die Herkunft ihrer Mittel öffendich Rechenschaft zu legen *. '2 Ebensowenig kann Isensee mit den Lehrern des Staatsrechts als Kronzeugen viel Staat machen. Mehr als ambivalent äußerte sich einse der Zeuge Maunz. Eincrseits qualifizierte dieser die Rechnungslegungspflicht als wnach ihrem Wortlaut unmitcelbar geltendes Rechr". Andererseits werde sie aber in der Praxis so gehandhabr, als bedürfe sie zu ihrer Akrualisierung cines Bundesgesetzes. ${ }^{3}$ Die übrigen Kommentatoren des Grundgeserzes von liberal bis konservativ, so sie nicht hierzu schweigen, räumen der Rechenschaftspflicht mehrheiclich Verfassungsstarus ein, addressiert an die Parteien, von gleichem Rang wie die Gründungsfreiheit und das Gebot innerparteilicher Demokrarie. ${ }^{4}$ Die Staarsrechtslehre beugt sich also keineswegs geschlossen unter das Joch der Isensee'schen Situationsjurisprudenz.

Nichr Mehrheit zählt bei der Auslegung von Gesetzen, kann Isensee kontern, sondern Wahrheit, jedenfalls Richtigkeit. Und die lässt sich, wenngleich in Gronzen, methodisch kontrollieren. Allein, Text, Kontext und Zweck der Pflicht zur öffentlichen Rechnungslegung sprechen gegen itue politisch interessierte Abstufung zum bloßen Auftrag. Der Wortlaut gibr, was selten genug vorkommt, eine deurliche Richtung an: Parteien nü̈ssen über die Herkunft und Verwendung ihrer Mitcel sowie über ihr Vermögen öffentlich Rechenschaft geben. « Dic Nachbarschaft zu Gründungsfrciheit und zum Gebor innerparteilicher Demokracie, also die Systemarik, spricht ebenso gegen Isensee wie die separatc Adresse an den Gesezzeber in Absar 3 des Artikels 21: „Das Nähere regeln Bundesgesetze. « Zum demokratischen Sinn und Zweck von Transparenz purde oben alles Wesentliche gesagc.

Kein ernstzunehmender Grundrechtsintergret käme auf die Idee, das Grundrecht auf Kriegsdienstverweigenung als bloßen Verfassungsauftrag zu lesen, nur weil das $\mathrm{Nä-}$ here durch Bundesgesetz zu regeln ist. Auch Isensee nicht. Die Sonderbehandlung der

II BVeriGE 2, 1/13

12 BVerfGE 20, 66/106; 24, 300/3 6; 85, 264/3181. (Hervorhebung niche im Original)

1) Maunz, in. Maunz/Dürig (Hg.), GG (1984), An. 21 Rn.78; F. Klcin, in: Schmidt-Blcibureu/K/ein, GG S.Aufl. (1995), Art. 2 I Rn. 16, meint unsicher, adic Rechenschaftelegungspslicht nach Art. 21 Abs, I Surz 4 ist an sech kein unmutulbar geliendes Reche " (Hervorh, niche im Orig.).

If Siche Preuß (Fn. 9); AKGG, Rn. 71; Morlok (Fn. 9); Drcier GG. An. 21 Fn. 10s If ; 1psen (Fn. 9), in: Sachs GG, Rn. $106 \mathrm{f}$. (nhoher Rang des Transparenzgebots $)$ ): Hoff́nann, DVBl. I gss', 836 (gegen Koesch DVBI. 1958, (99); Seifert (Fn. 9), Das Reche der politischen Parteien: Morlok, Zur Verfassungstheoric der Parteienfinanzierung, in: Mirteilungen des Instituts fur Deutsches und Europāisches Parcienrechı (1999), 6 ff. und ders., Spenden - Rechenschaft - Sankrionen, 76, (Are. 2 I / 4 als $\times$ verfassungsrecheliche Direktive «): Picrorh (Fn. 9), in: Jamss/Pieroth GG, Rn. 2 , (implizitc Ancrkennung ciner verfassungsunmitrelbaren Pflecle). Zur Kritik an Isensec vgl auch Bockenlöde, Dic Krise unserer Demokratic (Fn. 2). 
Rechenschafispflicht rechifertige er kurz und dunkel damit, dass Pflichten anders als

Rechte wunterhalb des Niveaus der Verfassung, im polirischen Prozess der demokratischen Geserzgebung festgelege « seien, dass aber »dic Festlegung von der Verfassung gesteuert" werde. Sehen wir uns also verfassungsmäßig verbürgte Pflichten an. Zum Beispiel die in die allgemeine Handlungsfreiheit eingelassene Pflicht, adie Rechte andereræ nicht zu verlerzen. Oder die nach Art. 6 II GG mzuvördersi (den Elrern) obliegende Pflichr ", ihre Kinder zu erziehen. Oder aber die von Art. s III GG allen Hochschullehrern aufgegebene "Treue zur Verfassung", auch eine Pflicht. Deren Verharmlosung zu cinfachgeseczlichen Verpflichtungen ist durch nichis veranlasst, obwohl stets Näheres der Regelung bedarf. Grocesk wird Isensees These durch den von ihm bemühten Vergleich zur Steuerpflicht. Zum einen felit $\mathrm{dcm}$ Grundgesecz, anders als hinsichtlich des expliziten Transparenzgebots, der Satz: *Die Bürger sind verpflichter, Steuern zu entrichren. « Zum anderen triffr die Steuerpflicht privates Vermögen; die Rechenschafrsplicht gründet dagegen im verfassungsrechtichen Sonderstatus der beträchtlich aus Sreuermitreln alimentierten Parteien und ihrer Rolle in der Demokratie. Nur weil die im argumentativen Noestand herbeibemühre Steuerpllichr kläglich hinkt, taugt sie dennoch nicht zum Vergleich. Kurzum: Der böse Schein entscehr, als sei die Degradienung des Transparenzgebots der begreiflichen Renitenz von Parteikadern geschuldet, eben diesem nachzukommen. So stever eine schlechte Praxis nicht einmal hinterrücks die Deutung des Grundgeserzes.

Isensces Verfassungsopfer auf dem Altar des Opportunen und seinc unerlaörte Milde gegenüber der für einen Bundeskanzler weiß Got» sgröblichen Missachtung von Recht und Gesecz $\propto$ kommen überraschend. Im System Isensee haben sie Methodc. Er verrät sie in einem Nebensacz: in einer Artacke gegen "jene Moralisten, die vor kurzem noch die begrenzte Regelverletzung öffentlich propagierten«. Natürlich weiß er, dass ziviler Ungehorsam, öffentlich geschehen, einsichtig sein und die vom Recht vorgesehenen Sanktionen aut sich nehmen muss," also mit dem Schattenreich der schwarzen Kassen nichts, aber auch gar nichts gemein liat. Warum also ziticrt Isensee den Bürgerprorest herbei, dem er in den Soer Jahren klirrend in die Parade fuhr und als Auswuchs eines »bio-pazifistischen Fundamentalismus«, als Bruch der bürgerlichen Friedenspflicht und des unbedingten Rechtsgehorsams geißelte? ${ }^{16}$ Erster Teil der Antwort: weil ihm jeder politische Moralismus zuwider ist, der sich erdreistet, mit demokratischen Argumenten einen Angriff auf das Herz des Staates zu uncernelimen. Er greift sters ein, wenn cr die "Staaclichkeit des Staatesu in Gefahr wähnt. Zweiter Teil: weil Isensee feinsinnig differcnziert zwischen Staatsträgern und Bürgern. Während er gegen letztere die ganze Majestät und Strenge von Recht und Verfassung in Anschlag bringt, bedient er die Staatsträger mit dem soft law des

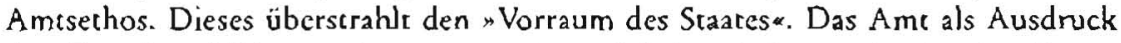
der Gemeinwohlidee "verlangt von seinem Inhaber Askese", damit "das Geserz herrschen kann*, so Isensee nebulös im Handbuch des Staacsrechts. ${ }^{77}$

Isensee hah um böflich zu formulieren, die Chance vertan, Parteien und Policiker energisch an eine rechtsstaatlich-demokratische Staaspraxis zu erinnern und beizurragen zu einer Rücktrigrskultur für alle Fälle, in denen Recht und Verfassung

1s Vgl. BVerfGE 73, 206/231 ff. und BayObLG JZ 1996, 404. G. Frankenberg, Liviler Ungehorsam und Rechtsstaatliche Demokratic. J7. $198_{4} .266 f f$. und ders., Der 7ivile Ungehorsan aisf dem Rerhesweg, in: Roth/Rucht (Hg.). Ncue Sorialc Bewegungen, 2. Aufl. (1991), s24ff. m. w. Nachow.

16 Vgl. J. Isensec, Ein Grundrecht auf Ungehorsam gegen das demokratische Gesetz? - Legutimation und Perversion des Widerstandsroches, in: B. Sereithofen ( $\mathrm{H}_{\mathrm{g}}$.). Frieden im L.ande (1983); ders., Widerstand gegen den technisechen Fortschritt, DOV $19^{8} 3$, $5^{6}$, ff. und ders., Dic Friedenspflicht der Bürger und das Gewaltmonopol des Staxies, 23 ff.

i7 J. Isensec, Stzat und Verfassung, in: HdbS R I, $1987, \$$ is. 
missachter werden. In der Gefahrenzone des Korruptionsverdachts sind strengere als nur amtsethische Maßstäbe anzulegen. Denn regierende wie auch nicht regierende Bürgerinnen und Bürger unterstehen dem gleichen Grundgeserz und den gleichen Gesetzen. Wo sie deren Regeln verletzen - als Bundespräsidenten, Bundeskanzler oder sonstige Inhaber öffenrlicher Ämter -, kommen Rechtsfolgen zum Zuge. Mit läppischen Entschuldigungen und semantischen Verrenkungen ist es nicho getan. Amtsträger haben die Wahl zwischen Rechtsgehorsam und Rücktritt. Auch sie becrifft die an alle potenziellen Schwarzfahrer in Frankfurter U- und Straßenbahnen gerichtete Mahnung: „Vergessen? Gilt nicht!»

Jens-Michael Priescer

Das Ende des

Züchtigungsrechts

Eine historische, dogmatische und straftheoretische Untersuchung

Das Zuchtigungsrecht der Eltern gehörte schon immer zu den rechtspolitischen Streithemen. Über lange Zeiten galt es als gewohnheitsrechtlich abgesichert. Erst mit dem Grundrecht der Kinder auf körperliche Unversehrtheit ergaben sich Probleme: den Befürwortern einer gesetzlichen Verankerung des Züchtigungsrechts erscheint jede Restriktion suspekt. Den Gegnern jedoch würde mit einer noch so restriktiven Verankerung die Hoffnung auf eine zukünftige Abschaffung zunichte gemacht.

Das Werk beleuchtet die Argumente gegen eine ausdrückliche Abschaffung, die nicht nur von Konservativen, sondern zum Teil auch von Kritikem des Strafrechts ausgingen, und untersucht, ob sich angesichts zal)/reicher Veränderungen des Rechtszustandes noch eine Basis findet, auf der sich das Zuchtigungsrecht begründen läßt. Überlegungen, ob eine mögliche Abschaffung straftheoretisch unbedenklich ist, runden das Werk ab.

2000, XX, 338 S., geb., 88,-DM, 642,- öS, 80,-sFr,

ISBN 3.7890-6045-3

(Strafrechtswissenschafi und Strafrechispolitik, Bd. 2)

NOMOS Verlagsgesellschaft

76520 Baden-Baden 